\title{
MÉTHOdE RAPIDE DE CONTROLE DE LA TENEUR EN RÉSIDUS DE QUELQUES PESTICIDES ORGANOCHLORÉS DANS LES PRODUITS LAITIERS
}

\author{
par \\ J. GOURSAUD, F. M. LUQUET et J. CASALIS \\ Laboratoire d'industrie laitière de Douai
}

La méthode de dosage des résidus de pesticides organochlorés décrite ci-dessous, est une variante de celle proposée par Langlois, Stemp \& Liska. Appliquée couramment au Laboratoire d'Industrie Laitière de Douai, elle permet d'obtenir rapidement des résultats précis et reproductibles.

\section{Principe de la méthode}

Les pesticides organochlorés sont des composés très stables chimiquement. Ils sont liposolubles et s'accumulent donc dans les matières grasses. Leur dosage est effectué par chromatographie en phase gazeuse au moyen d'un chromatographe avec détecteur à capture d'électrons.

\section{2. Équipement}

\section{A. Appareils.}

- Chromatographe avec détecteur à capture d'électrons, type Aerograph, modèle 600 D, ou Hy-Fi III, modèle 1200 , d'électrons,

- Enregistreur Honeywell simple piste, 1 mvolt,

- Evaporateur rotatif sous vide, type Rotavapor Büchi, Technique.

- Colonne à distiller, à remplissage statique, type Verre et

\section{B. Verrerie.}

- Colonnes de chromatographie, en verre Pyrex de $100 \mathrm{~cm}$ de longueur, à robinet plat, diamètre $2 \mathrm{~cm}$, 
- Béchers de $200 \mathrm{ml}$, entonnoirs, ballons forme poire à cols rodés, de $500 \mathrm{ml}$,

- Fioles calibrées de 5, 10, 20, 25, 50, $100 \mathrm{ml}$.

\section{Réactifs.}

- Ether de pétrole $40-60^{\circ}$, chlorure de méthylène, hexane, sulfate de sodium anhydre, florisil 60/100 Mesh,

- Pesticides étalons à l'état pur : HCH, lindane, heptachlore, aldrine, heptachlore époxyde, dieldrine, DDT...

\section{Mode opératoire}

\section{A. Contrôle de la verrerie et des réactifs.}

10 Contrôle de la propreté de la verrerie.

La chromatographie en phase gazeuse nécessite un parfait état de propreté de la verrerie : une machine à laver automatique et programmée, dans laquelle le rinçage final de la vaisselle est fait à l'eau déminéralisée, donne à cet égard, d'excellents résultats. Toute la verrerie sera, avant emploi, rincée avec un éluant obtenu par mélange de 65 p. 100 d'éther de pétrole et 35 p. 100 de chlorure de méthylène, ces deux produits ayant été préalablement distillés.

$2^{\circ}$ Contrôle de la pureté des réactifs.

Il est indispensable avant chaque série d'analyses, de purifier et de contrôler la pureté de tous les réactifs.

a) Solvants.

Distiller sur colonne à remplissage statique l'éther de pétrole, le chlorure de méthylène et l'hexane;

Eliminer la tête et la queue de distillation;

Contrôler la pureté de chacun des solvants par une analyse chromatographique effectuée dans les conditions précisées ci-dessous

b) Florisil.

Répartir le florisil dans des mortiers sur une épaisseur de $1 \mathrm{~cm}$;

Passer à l'étuve à $140^{\circ} \mathrm{C}$ pendant $14 \mathrm{~h}$, pour activer le produit ;

Après refroidissement, ajouter 5 p. 100 d'eau en poids, homogénéiser soigneusement;

Conserver dans des bouteilles en verre de couleur foncée, à l'abri de la lumière. Le produit est prêt à l'emploi au bout de quelques heures;

Contrôler la pureté par une analyse chromatographique effectuée dans les conditions précisées ci-dessous. 


\section{c) Sulfate de sodium.}

Contrôler la pureté par analyse chromatographique effectuée dans les conditions précisées ci-dessous.

\section{B. Extraction des résidus de pesticides.}

10 Chromatographie préparative sur colonne de florisil.

a) Préparation de la colonne de florisil.

Garnir la partie inférieure de la colonne d'un tampon de laine de verre ou de coton hydrophile ; y verser environ $120 \mathrm{ml}$ d'éluant.;

Peser $35 \mathrm{~g}$ de florisil dans un bécher, bien mouiller avec l'éluant, homogénéiser avec un agitateur. Introduire au moyen d'un entonnoir le produit ainsi préparé dans la colonne; le tasser de telle sorte que l'éluant se trouve au-dessus du florisil ; chasser les bulles d'air incluses en faisant rouler la colonne entre les paumes des mains. Ajouter $5 \mathrm{~g}$ de sulfate de sodium. Eluer dans la colonne $50 \mathrm{ml} \mathrm{du}$ mélange éther de pétrole-chlorure de méthylène qui seront éliminés.

b) Préparation des échantillons.

b 1 : Peser dans un bécher $25 \mathrm{~g}$ de florisil. Ajouter $80 \mathrm{ml}$ d'éluant. Homogénéiser en agitant pendant $3 \mathrm{mn}$ au moyen d'un agitateur de verre. Laisser reposer et décanter le solvant en excès.

b 2 : Peser à $0,5 \mathrm{mg}$ près dans un bécher une fraction du produit à analyser de telle sorte que le poids de matière grasse soit compris entre 300 et $900 \mathrm{mg}$, soit :

\begin{tabular}{|c|c|}
\hline Pour le lait entier ..... & $10 \mathrm{~g}$ \\
\hline Pour la crème ... & $2 \mathrm{~g}$ \\
\hline Pour le beurre ....... & \\
\hline Pour le fromage.... & 1 à $2 \mathrm{~g}$ \\
\hline
\end{tabular}

Lait entier et crème. - Introduire dans le bécher contenant l'échantillon les $25 \mathrm{~g}$ de florisil préparé de la manière indiquée ci-dessus. Homogénéiser soigneusement. Ajouter $25 \mathrm{ml}$ d'hexane distillé. Homogénéiser à l'aide d'un agitateur de verre.

Beurre. - Introduire dans le bécher contenant l'échantillon, $25 \mathrm{ml}$ d'hexane distillé. Homogénéiser soigneusement. Ajouter les $25 \mathrm{~g}$ de florisil préparé de la manière indiquée ci-dessus. Homogéniser à l'aide d'un agitateur de verre.

Fromage. - Introduire dans le bécher contenant l'échantillon, $25 \mathrm{ml}$ d'hexane distillé. Homogénéiser avec un agitateur mécanique à hélice. Rincer l'agitateur avec $300 \mathrm{ml}$ de solvant d'élution. Ajouter les $25 \mathrm{~g}$ de florisil préparé de la manière indiquée ci-dessus. Homogénéiser.

c) Extraction des pesticides.

Verser dans la colonne préparée suivant a), le mélange échantillon + hexane + florisil. Tasser en évitant l'inclusion de bulles 
d'air. Ajouter $5 \mathrm{~g}$ de sulfate de sodium. En utilisant une quantité déterminée de solvant (300 à $500 \mathrm{ml})$, éluer goutte à goutte dans un ballon en forme de poire et à col rodé.

Lorsque le volume d'élution est atteint, évaporer à sec au moyen d'un évaporateur rotatif sous vide. Le bain-marie doit être réglé à une température de $30^{\circ} \mathrm{C} \pm 2^{\circ} \mathrm{C}$.

Après évaporation complète du solvant, les pesticides s'il y en a, sont rassemblés au fond du ballon en cercles concentriques réguliers. Dissoudre ces résidus par une quantité connue d'hexane distilié (5 ou $10 \mathrm{ml})$.

Effectuer toujours une série d'analyses avec le même lot de florisil préparé comme indiqué au paragraphe 3. Chaque lot sera repéré par un numéro.

Pour chaque série d'analyses, préparer une colonne de florisil, identique à celle utilisée pour l'analyse d'un produit laitier. Cette colonne "à blanc ", sera éluée dans les mêmes conditions que les colonnes expérimentales, mais l'échantillon laitier sera remplacé par une solution de pesticide de concentration connue.

Cette solution aura été soumise au préalable à une analyse chromatographique, et l'on comparera les chromatogrammes obtenus avant et après élution sur colonne de florisil. Les pics obtenus après élution sur colonne, sont toujours inférieurs ou au maximum égaux, aux pics obtènus avant élution. Si besoin est, calculer le pourcentage de rétention, pour chaque catégorie de pesticide, du lot de florisil utilisé.

En tenir compte dans le calcul quantitatif.

\section{Etablissement des courbes d'étalonnage pour les différents pesticides.}

10 Préparation de solutions étalons.

Dissoudre dans une fiole jaugée de $100 \mathrm{ml}, 100 \mathrm{mg}$ de pesticide dans de l'hexane distillé.

Par dilutions successives de cette solution mère, préparer une gamme de solutions de concentrations connues du pesticide considéré $\left(1 / 25000^{\mathrm{e}}\right.$ à $1 / 200000^{\mathrm{e}}$ pour $\mathrm{l}^{\prime} \mathrm{HCH}$ par exemple).

\section{$2^{\circ}$ Analyse des solutions étalons.}

Les conditions de l'analyse doivent être rigoureusement constantes.

Dimensions de la colonne en verre Pyrex : longueur : 5 pieds, soit $1,50 \mathrm{~m}$; diamètre : $1 / 8$ de pouce, soit $3,17 \mathrm{~mm}$.

Température de la colonne : 180 à $195^{\circ} \mathrm{C}$.

Débit en sortie de détecteur : 45 à $50 \mathrm{ml}$, par minute, réglé par un débit constant d'azote. 


\title{
CHROMATOGRAMME 1
}

\author{
COLONNE PYREX $1,50 \mathrm{~m}$ \\ garnie DOW $115 \%$ sur chromosorb W
}

$I$
5
$I$
1
5
0
-1

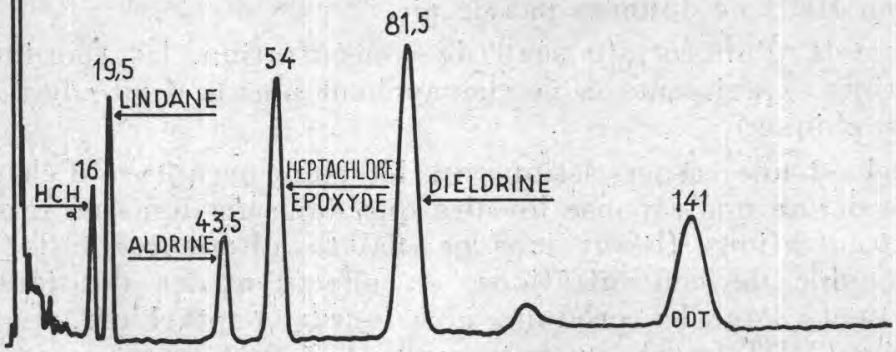

INJECTIONS DE $5 \mu$ I

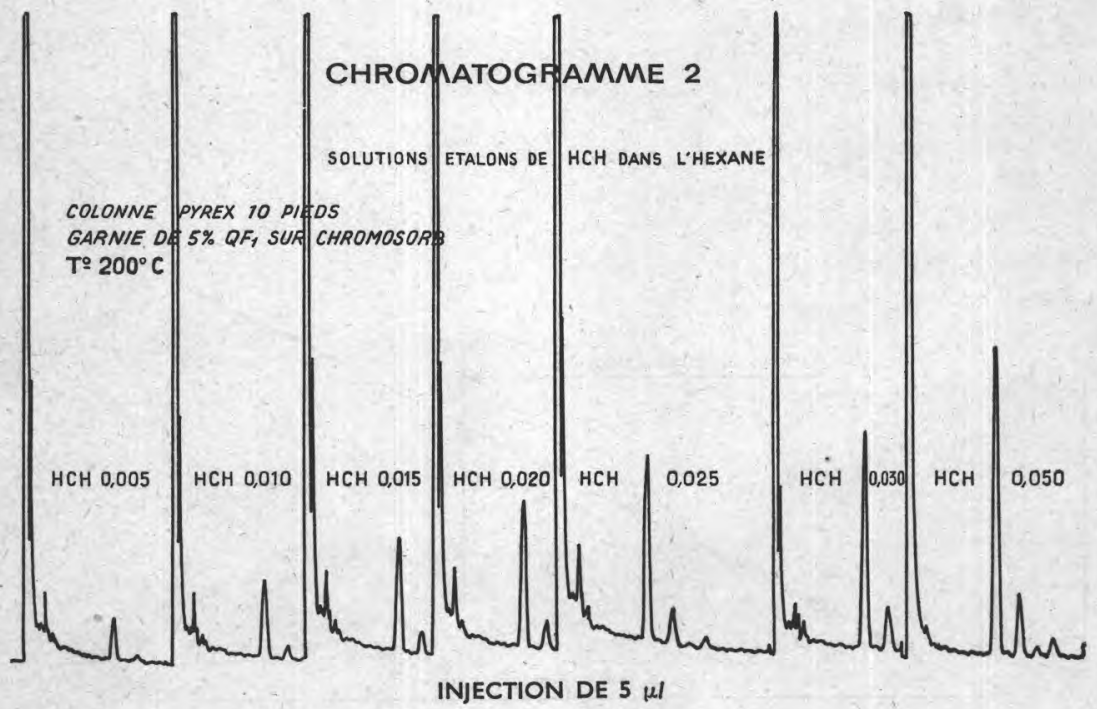


Volume d'échantillon injecté en utilisant toujours la même micro seringue : $5 \mu l$.

Pour les produits à temps de rétention court : HCH, heptachlore, aldrine (v. chromatogramme 1), on obtient des pies dont la hauteur est proportionnelle à la concentration. Il faut tracer la courbe :

$$
h=f(c)
$$

Par exemple, injecter dans le chromatographe $5 \mu l$ de chacune des solutions étalons en HCH, en commençant par la concentration la plus faible, de façon à obtenir une série de pics de hauteur variable ( $v$. chromatogramme 2).

Porter sur un graphique les hauteurs en fonction des concentrations : les points obtenus se répartissent régulièrement sur une droite passant par l'origine, car une solution de concentration nulle en $\mathrm{HCH}$ ne donnera pas de pic.

Au-delà d'un certain seuil de concentration, les coordonnées des points expérimentaux ne chevauchent plus la droite de départ. (Cf. graphique.)

Ceci est une caractéristique du détecteur à capture d'électrons qui ne donne une réponse linéaire que dans un domaine restreint de concentrations. Il faut dans les analyses ultérieures, rester dans ce domaine de concentrations, en effectuant les dilutions qui s'imposent. Pour les produits plus lourds, heptachlore, epoxyde, dieldrine, DDT (v. chromatogramme 1), il faut tracer la courbe : surface du pic en fonction de la concentration.

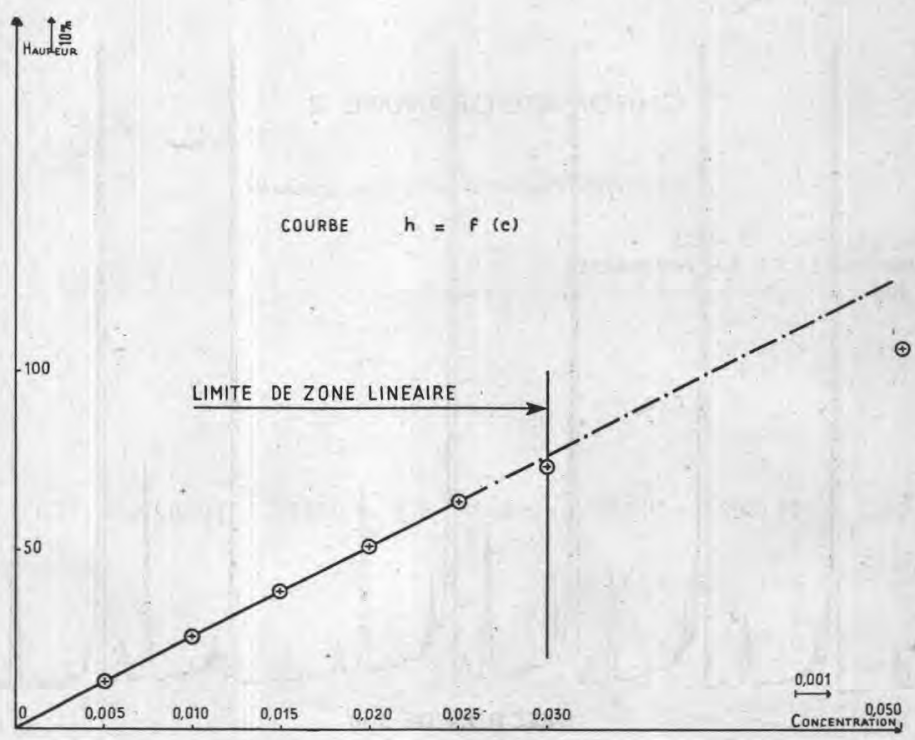




\title{
CHROMATOGRAMME 3
}

\author{
FROMAGE CONTAMINE
}

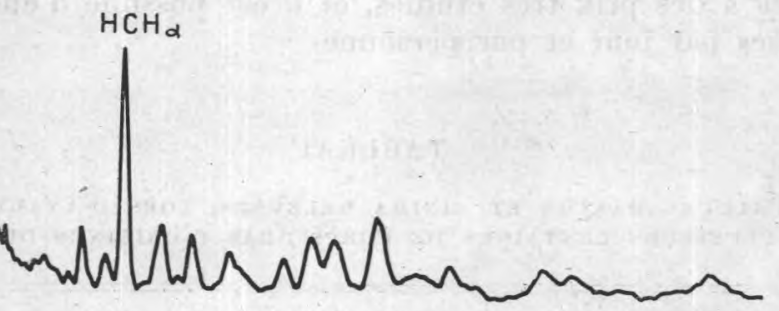

INJECTION DE $5 \mu$ I

D. Analyse de l'échantillon (chromatogramme 3).

Injecter la solution préparée et calibrée $(5$ ou $10 \mathrm{ml})$, dans le chromatographe, à l'aide de la seringue qui a servi à dresser la courbe étalon.

Le contrôle qualitatif des pesticides se fera par la mesure des temps de rétention. Il est bon pour vérifier une analyse, de travailler sur deux colonnes chromatographiques différentes, dans des conditions expérimentales différentes.

Par exemple :

- Chromatographe 600 , équipé d'une colonne 5 pieds, garnie de phase stationnaire QF 1 sur chromosorb, température $195^{\circ}$. 
- Four 550, équipé d'une colonne 10 pieds, garnie de phase stationnaire DOW 11 sur chromosorb, température $180^{\circ} \mathrm{C}$.

Le contrôle et la vérification qualitatifs se font en injectant l'échantillon dans les deux colonnes de polarité différentes, et par comparaison avec les injections des solutions étalons.

Pour les qualifications les plus délicates, il est possible d'injecter en une seule fois, une partie aliquote de l'échantillon à analyser, et une solution étalon du pesticide présumé.

Le dosage quantitatif se fera par comparaison de la hauteur ou de la surface du pic, avec la courbe étalon. Avoir soin de rester dans la gamme de linéarité de l'appareil, en effectuant au besoin, des dilutions de la solution à analyser.

Les résultats peuvent être donnés en $\mathrm{mg}$ de pesticide par $\mathrm{kg}$ de produit, c'est-à-dire en ppm.

\section{Prix de revient}

En tenant compte :

- de l'amortissement du matériel : chromatographe, colonne en Pyrex pour four de chromatographe, source radioactive;

- des produits à utiliser : florisil, éluant, hexane, azote, phase stationnaire pour colonne,

on arrive à des prix très étudiés, et il est possible d'effectuer 7 à 8 analyses par jour et par personne.

\section{TABLEAU}

Valeurs maxima et minima Relevées, lors D'analyses DE PLUSIEURS CENTAINES DE CORPS GRAS D'ORIGINES DIVERSES

\begin{tabular}{|c|c|c|c|c|c|c|}
\hline $\begin{array}{c}\text { Teneur } \\
\text { en } H C H\end{array}$ & $\begin{array}{c}\text { Lait } \\
\text { entier }\end{array}$ & Crème & Beurre & Fromage & Suif & $\begin{array}{l}\text { Graisse } \\
\text { de poulet }\end{array}$ \\
\hline Maxi ... & $0,045 \mathrm{ppm}$ & $0,60 \mathrm{ppm}$ & $3,40 \mathrm{ppm}$ & $0,90 \mathrm{ppm}$ & $10 \mathrm{ppm}$ & $33 \mathrm{ppm}$ \\
\hline Mini...... & $0,005 \mathrm{ppm}$ & $0,40 \mathrm{ppm}$ & $0,10 \mathrm{ppm}$ & $0,001 \mathrm{ppm}$ & $1 \mathrm{ppm}$ & $0,10 \mathrm{ppm}$ \\
\hline
\end{tabular}

\section{Résumé}

L'analyse des résidus de pesticides par chromatographie en phase gazeuse est aujourd'hui une opération qui peut être conduite couramment en laboratoire. 
La méthode proposée est une variante de celle de Langlois, Stemp \& Liska. Ses avantages principaux sont la rapidité et la sensibilité de la technique d'analyse.

Les résultats trouvés montrent que, selon les matières grasses, les teneurs en résidus de $\mathrm{HCH}$ sont très variables.

\section{Summary}

The analysis of pesticide residues by means of gas chromatography can nowadays be practised currently by laboratories.

The proposed method is a variation of the Langlois, Stemp \& Liska method. Its main advantages are rapidity and sensibility of analysis techniques.

The findings show, according to the fats, most fluctuating $\mathrm{HCH}$ residue contents.

\section{BIBLIOGRAPHIE}

Langlots (B. E.), Stemp (A. R.) \& Liska (B. J.). Rapid clean up of dairy products for analysis of chlorinated insecticide residue by electron capture gas chromatography. Agricultural and Food Chemistry, vol. 12, no 3, May-June 1964.

Moats (W. A.). One step chromatographic clean up of ehlorinated hydrocarbon pesticide residues in butterfat. Chromatography on florisil. Journal of the A.O.A.C., vol. $46, \mathrm{n}^{\circ} 2,1963$.

Thieulin (G.), Pantaleon (J.), Richou (L.) \& Cumont (G.). Les résidus de pesticides dans le lait et les produits laitiers. Bulletin de l'Académie nationale de Médecine, tome 150, no 24-25.

Mestres (R.), Barthes (F.) \& Mlle Priu (M.) (Faculté de Pharmacie), Portal (E.) (Laboratoire de la Répression des Fraudes). Recherche et. dosage de l'aldrine, de la dieldrine et du DDT dans le lait et le beurre. Société de Pharmacie de Montpellier, séance du 30-6-1966.

MCCULLY (K. A.) \& McKinley (W. P.). Determination of chlorinated pesticide residues in fat by electron capture gas chromatography. Journal of the A.O.A.C., vol, $47, \mathrm{n}^{\circ} 4,1964$.

KLEIN (A. K.), WATtS (J. O.) \& RAMTCO (J. N.). Electron capture gas chromatography for determination of DDT in butter and some vegetable oils. Journal of the A.O.A.C., vol. 46, no 2, 1963. 